\title{
Aetiology of Bacteraemia as a Risk Factor for Septic Shock at the Onset of Febrile Neutropaenia in Adult Cancer Patients
}

\author{
Regis Goulart Rosa and Luciano Zubaran Goldani \\ Infectious Diseases Unit, Infectious Diseases Division of Hospital de Clínicas de Porto Alegre, \\ Universidade Federal do Rio Grande do Sul, Ramiro Barcelos 2350, Room 700, Porto Alegre 90640-000, RS, Brazil \\ Correspondence should be addressed to Luciano Zubaran Goldani; lgoldani@ufrgs.br
}

Received 11 November 2013; Accepted 21 February 2014; Published 20 March 2014

Academic Editor: Ketoki Kapila

Copyright (c) 2014 R. G. Rosa and L. Z. Goldani. This is an open access article distributed under the Creative Commons Attribution License, which permits unrestricted use, distribution, and reproduction in any medium, provided the original work is properly cited.

Septic shock (SS) at the onset of febrile neutropaenia (FN) is an emergency situation that is associated with high morbidity and mortality. The impact of the specific aetiology of bloodstream infections (BSIs) in the development of SS at the time of FN is not well established. The aim of this study was to evaluate the association between the aetiology of BSIs and SS at the time of FN in hospitalised adult cancer patients. This prospective cohort study was performed at a single tertiary hospital from October 2009 to August 2011. All adult cancer patients admitted consecutively to the haematology ward with FN were evaluated. A stepwise logistic regression was conducted to verify the association between the microbiological characteristics of BSIs and SS at the onset of FN. In total, 307 cases of FN in adult cancer patients were evaluated. There were 115 cases with documented BSI. A multivariate analysis showed that polymicrobial bacteraemia $(P=0.01)$ was associated with SS. The specific blood isolates independently associated with SS were viridans streptococci $(P=0.02)$ and Escherichia coli $(P=0.01)$. Neutropaenic cancer patients with polymicrobial bacteraemia or BSI by viridans streptococci or Escherichia coli are at increased risk for SS at the time of FN.

\section{Introduction}

Despite improvements in treating febrile neutropaenia (FN) and sepsis over the past decade, septic shock (SS) continues to be associated with substantial morbidity and mortality among cancer patients undergoing intensive cytotoxic chemotherapy [1]. The unpredictable clinical course of infections in neutropaenic patients because of the lack of an adequate inflammatory response makes managing $\mathrm{FN}$ a significant challenge because clinically stable patients may suddenly progress to severe sepsis or SS [2].

SS is a result of the host response to the pathogen and is dependent on the virulence of the microorganism and the infection site [3]. The known risk factors for SS in immunocompetent patients include advanced age, low functional status, and the presence of cancer, clinical comorbidities, nosocomial infections, and infection that does not originate in the urinary tract [4-6]. Infection with certain bacteria, such as Staphylococcus aureus and Pseudomonas aeruginosa, is also associated with an increased risk for SS, as the expression of certain proteins or molecules (virulence factors) contributes to pathogen replication and dissemination by subverting or eluding the host's defences [7]. Unfortunately, data regarding the influence of microbiological factors on the development of SS in cancer patients with FN is scarce. Therefore, we conducted a study with the aim of evaluating the association between microbiological aspects of bloodstream infections (BSIs) and SS development at the onset of FN in hospitalised adult cancer patients.

\section{Methods}

2.1. Study Design and Participants. A prospective cohort study was conducted at a single referral centre for adult bone marrow transplantation in Southern Brazil from October 2009 to August 2011. This study followed all consecutive haemodynamically stabile cancer patients older than 18 years of age who were admitted to the haematology ward of the Hospital de Clínicas de Porto Alegre (Porto Alegre, Brazil) with neutropaenia (i.e., an absolute neutrophil count (ANC) $<500$ cells $/ \mathrm{mm}^{3}$ or $<1000$ cells $/ \mathrm{mm}^{3}$ with an expectation of a decrease to $<500$ cells $/ \mathrm{mm}^{3}$ during the ensuing $48 \mathrm{~h}$ ). 
The subjects who developed fever (i.e., a single axillary temperature measurement $\geq 38.5^{\circ} \mathrm{C}$ or sustained temperature $\geq 38.0^{\circ} \mathrm{C}$ over a $1 \mathrm{~h}$ period) during the course of neutropaenia were entered into the study. Outpatients, patients who had neutropaenia caused by a specific aetiology other than an adverse reaction to chemotherapy, and patients who had episodes of FN without documented bacteraemia were excluded. Subjects were allowed to reenter the study after an initial episode of FN if they remained free of signs or symptoms of infection for at least 7 days after completing the treatment for the first episode and if all causative organisms, if any, were eradicated.

2.2. Definitions. Microbiological studies, which included 2 separate blood samples that were obtained from 2 different anatomical sites for culture, were performed at the onset of fever, according to standard practice. In the absence of an indwelling central venous catheter, 2 blood samples were obtained from 2 distinct peripheral veins. When an indwelling central venous catheter was present, 1 blood sample was obtained through this catheter, and a second sample was obtained from a peripheral vein. The susceptibilities of the isolated pathogens to antibiotics were evaluated according to the recommendations of the Clinical and Laboratory Standards Institute [8]. Bacteraemia caused by coagulase-negative Staphylococcus spp. was diagnosed after 2 positive results from 2 independent cultures. Bacteraemia indicated by 1 positive culture was considered to be diagnostic for the other microorganisms. Polymicrobial BSI was characterised as a bacteraemic episode due to at least two different pathogens isolated from the same blood sample. Multidrug-resistant (MDR) bacteraemia was defined as a BSI with methicillinresistant staphylococci or vancomycin-resistant enterococci for Gram-positive bacteria or as resistance to $\geq 3$ classes of antimicrobial agents for Gram-negative bacteria. Clinical comorbidity was determined by the presence of heart failure, diabetes mellitus, chronic pulmonary disease, chronic liver disease, or chronic renal failure. Profound neutropaenia was characterised by an ANC $<100$ cells $/ \mathrm{mm}^{3}$. The patients were divided into 2 groups based on their chemotherapy regimens: a high-dose chemotherapy group that included patients who underwent haematopoietic stem cell transplantation or induction chemotherapy and a standard-dose chemotherapy group that included patients who underwent consolidation or maintenance chemotherapy. Nosocomial-acquired FN was defined as the onset of FN after 48 hours of hospitalisation. The oral mucositis grade was classified according to the World Health Organisation's oral toxicity scale [9].

2.3. Outcomes and Followup. The primary outcome measure of the present study was SS at the onset of fever in neutropaenic patients. SS was defined as persistent haemodynamic instability (systolic arterial pressure $<90 \mathrm{mmHg}$ or a reduction in systolic blood pressure $>40 \mathrm{mmHg}$ from baseline) despite adequate fluid resuscitation $(30 \mathrm{~mL}$ per $\mathrm{Kg}$ of crystalloid) with at least 2 systemic inflammatory response syndrome criteria [10]. The secondary outcome was mortality by day 28 . Researchers who were not associated with the assistant physician's team conducted the patient followups through interviews and medical record reviews using a standardised data collection instrument. The followup was maintained for 28 days after the onset of fever in the neutropaenic patients. For the subjects who were discharged before 28 days, follow-up telephone calls were made on the 28th day after the onset of FN to determine whether they remained alive; if a patient was deceased at the time of the phone call, the survival time was calculated based on the date of death reported by the family.

2.4. Statistical Analysis. Stepwise logistic regression analysis was performed to determine whether the microbiological characteristics of BSIs were risk factors for SS at the time of FN. All clinical and microbiological variables that had a $P$ value $<0.10$ in the univariate analysis were included. In the multivariate model, independent variables were eliminated from the highest to the lowest $P$ value but remained in the model if the $P$ value was $<0.05$. Odds ratios (OR) were estimated with $95 \%$ confidence intervals (95\% CI). Kaplan-Meier curves were utilised to evaluate the timedependent occurrence of death; the log-rank test was applied for between-group comparisons. The statistical analysis was performed using STATA version 12 (Stata Corp LP, USA).

2.5. Ethics Statement. Written informed consent was obtained from all study participants. The institutional review board of the Hospital de Clínicas de Porto Alegre approved the study.

\section{Results}

In total, 307 episodes of FN (in 169 patients) were evaluated; a total of 115 BSIs were documented (37.4\% of all episodes). Antibiotic prophylaxis was not administered to any patient. The incidence of SS was 14.7\% (17 episodes).

The characteristics of the study population and the specific pathogens responsible for all BSIs in the present cohort are shown in Table 1. Subjects with haematological malignancies comprised $83.5 \%$ of the study population; haematopoietic stem cell transplantation was performed in $21.7 \%$ of the cases. Forty-eight percent of the study sample had some degree of chemotherapy-induced mucositis. The proportion of nosocomial episodes of FN was $88.7 \%$. In descending order, the predominant blood isolates were Escherichia coli, coagulase-negative staphylococci, Klebsiella pneumoniae, Pseudomonas aeruginosa, viridans streptococci, and Enterococcus spp. Among all BSIs evaluated, 38 cases were due to MDR bacteria (Table 2): 4 cases in the SS group $(23.5 \%)$ and 34 cases in the non-SS shock group (34.6\%). Methicillin resistance and the production of extendedspectrum beta-lactamase were the most frequent types of antimicrobial resistance, occurring in $96.2 \%$ of BSIs involving Gram-positive MDR bacteria and $83.3 \%$ of BSIs involving Gram-negative MDR bacteria, respectively.

A univariate analysis revealed that polymicrobial BSI $(P=0.01)$ and bacteraemia by Escherichia coli $(P=0.04)$ were associated with the main outcome (Table 3 ). Multidrugresistant (MDR) bacteraemia was not associated with SS at 
TABLE 1: Study population characteristics and microorganisms isolated in 115 cases of febrile neutropenia (FN) in hospitalised cancer patients with documented bloodstream infection.

\begin{tabular}{|c|c|}
\hline Age, mean years \pm SD & $42.9 \pm 14.1$ \\
\hline Female sex & $52(45.2)$ \\
\hline \multicolumn{2}{|l|}{ Type of cancer } \\
\hline Acute myeloid leukaemia & $59(51.3)$ \\
\hline Acute lymphoblastic leukaemia & $19(16.5)$ \\
\hline Chronic myeloid leukaemia & $7(6.1)$ \\
\hline Multiple myeloma & $11(9.6)$ \\
\hline Lymphoma & $15(13.0)$ \\
\hline Other solid tumours & $4(3.5)$ \\
\hline Relapsing underlying disease & $59(51.3)$ \\
\hline Clinical comorbidity & $36(31.3)$ \\
\hline \multicolumn{2}{|l|}{ Phase of chemotherapy } \\
\hline Induction & $27(23.5)$ \\
\hline Consolidation & $37(32.2)$ \\
\hline Maintenance & $26(22.6)$ \\
\hline HSCT & $25(21.7)$ \\
\hline \multicolumn{2}{|l|}{ Oral mucositis } \\
\hline Without oral mucositis & $59(51.3)$ \\
\hline Grade I & $33(28.7)$ \\
\hline Grade II & $10(8.7)$ \\
\hline Grade III & $6(5.2)$ \\
\hline Grade IV & $7(6.1)$ \\
\hline $\begin{array}{l}\text { ANC at the time of diagnosis of } \mathrm{FN} \\
\text { mean cells } / \mathrm{mm}^{3} \pm \mathrm{SD}\end{array}$ & $206.1 \pm 218.5$ \\
\hline $\begin{array}{l}\text { Profound neutropenia at the time } \\
\text { of diagnosis of } \mathrm{FN}^{*}\end{array}$ & $52(45.2)$ \\
\hline Nosocomial-acquired episodes of FN & $102(88.7)$ \\
\hline \multicolumn{2}{|l|}{ Bloodstream isolates $^{\dagger}$} \\
\hline Escherichia coli & 48 (41.7) \\
\hline Coagulase-negative staphylococci & $36(31.3)$ \\
\hline Klebsiella pneumonia & $13(11.3)$ \\
\hline Pseudomonas aeruginosa & $11(9.5)$ \\
\hline Viridans streptococci & $8(6.9)$ \\
\hline Enterococcus spp. & $4(3.4)$ \\
\hline Serratia spp. & $2(1.7)$ \\
\hline Enterobacter spp. & $2(1.7)$ \\
\hline Candida spp. & $2(1.7)$ \\
\hline Salmonella spp. & $1(0.8)$ \\
\hline Staphylococcus aureus & $1(0.8)$ \\
\hline Kocuria varians & $1(0.8)$ \\
\hline
\end{tabular}

Data presented as $n$ (\%) unless otherwise indicated. SD: standard deviation; ANC: absolute neutrophil count; HSCT: hematopoietic stem cell transplantation; *ANC $<100$ cells $/ \mathrm{mm}^{3}$; ${ }^{\dagger}$ There were 12 cases of polymicrobial bloodstream infections.

the onset of FN with either the Gram-positive MDR or Gramnegative MDR bacteria.

After multiple logistic regression analyses were performed (Table 4 , model 1 ), the only variable that constituted an independent risk factor for SS at the time of FN was
TABLE 2: Multidrug-resistant bacteria isolated in 38 cases of bacteraemia in febrile neutropenic patients.

\begin{tabular}{lc}
\hline Microorganism & Number isolated (\%) \\
\hline Gram-positive & \\
MR coagulase-negative staphylococci & $25(65.7)$ \\
MR Staphylococcus aureus & $1(2.6)$ \\
VR Enterococcus faecalis & $1(2.6)$ \\
Gram-negative & \\
Escherichia coli ESBL & $7(18.4)$ \\
Klebsiella pneumoniae ESBL & $3(7.8)$ \\
Enterobacter spp. & $1(2.6)$ \\
Serratia spp. & $1(2.6)$ \\
\hline
\end{tabular}

MR: methicillin resistant; VR: vancomycin resistant; ESBL: extendedspectrum beta-lactamase. There was 1 case of polymicrobial multidrugresistant bacteraemia.

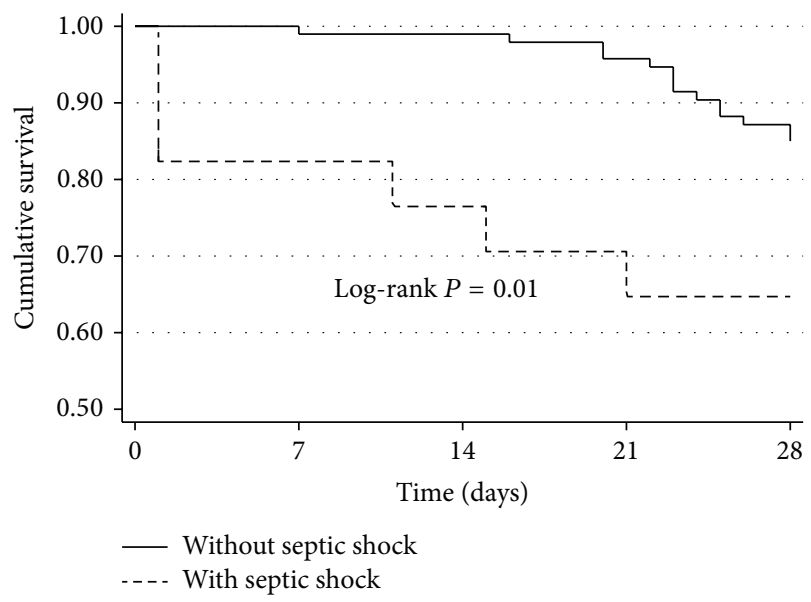

FIGURE 1: Survival curves according to the presence of septic shock at the time of febrile neutropenia in hospitalised adult cancer patients.

polymicrobial BSI (OR, 5.41, 95\% CI, 1.48-19.79). A second logistic regression model was used to assess the effect of specific pathogens on the development of SS without the inclusion of other microbiological variables (Table 4, model 2). This model was conducted to avoid the dilution of the effect of specific pathogens by other microbiological factors in the multivariate analysis. The specific blood isolates that were independently associated with the main outcome were viridans streptococci (OR, 7.58, 95\% CI, 1.34-42.80) and Escherichia coli (OR, 4.30, 95\% CI, 1.34-14.48). The percentage of the polymicrobial samples that included E. coli and viridans streptococci was $58.3 \%$ (7 cases) and 25\% (3 cases), respectively.

As expected, the 28-day mortality rate of the patients who presented with SS at the time of FN was greater than that of the patients who did not present with SS (35.2\% versus $14.2 \%$, $\log$-rank $P=0.01$ ) (Figure 1).

\section{Discussion}

The present prospective cohort study demonstrated that cancer patients with polymicrobial bacteraemia were more 
TABLE 3: Univariate analysis of the risk factors for septic shock (SS) at the time of febrile neutropenia (FN) in hospitalised cancer patients.

\begin{tabular}{|c|c|c|c|c|}
\hline Variable & SS group $(n=17)$ & Non-SS group $(n=98)$ & OR $(95 \% \mathrm{CI})$ & $P$ value \\
\hline Age, years, mean \pm SD & $43.4 \pm 16.0$ & $42.8 \pm 13.8$ & $1.00(0.96-1.04)$ & 0.87 \\
\hline Female sex & $10(58.8)$ & $42(42.8)$ & $1.90(0.66-5.41)$ & 0.22 \\
\hline Haematological neoplasm & $13(76.4)$ & $83(84.6)$ & $0.58(0.16-2.04)$ & 0.40 \\
\hline Relapsing underlying disease & $10(58.8)$ & $49(50.0)$ & $1.42(0.50-4.05)$ & 0.50 \\
\hline Clinical comorbidity & $2(11.7)$ & $34(34.6)$ & $0.25(0.05-1.16)$ & 0.07 \\
\hline High-dose chemotherapy regimens* & $5(29.4)$ & $47(47.9)$ & $0.45(0.14-1.38)$ & 0.16 \\
\hline \multicolumn{5}{|l|}{ Oral mucositis } \\
\hline Grade I & $4(23.5)$ & $29(29.5)$ & $0.76(0.21-2.71)$ & 0.68 \\
\hline Grade II & $2(11.7)$ & $8(8.1)$ & $1.38(0.25-7.63)$ & 0.70 \\
\hline Grade III & $1(5.8)$ & $5(5.1)$ & $1.11(0.11-10.66)$ & 0.92 \\
\hline Grade IV & $1(5.8)$ & $6(6.1)$ & $0.92(0.09-8.63)$ & 0.94 \\
\hline ANC at the time of diagnosis of FN, mean \pm SD & $161.7 \pm 219.0$ & $213.8 \pm 218.7$ & $0.99(0.99-1.00)$ & 0.36 \\
\hline Profound neutropenia at the time of diagnosis of $\mathrm{FN}^{\dagger}$ & $9(52.9)$ & $43(43.8)$ & $1.43(0.51-4.04)$ & 0.49 \\
\hline BSI involving Gram-positive bacteria & $6(39.2)$ & $40(40.8)$ & $0.79(0.27-2.31)$ & 0.66 \\
\hline BSI involving Gram-negative bacteria & $14(82.3)$ & $60(61.2)$ & $2.96(0.79-10.97)$ & 0.10 \\
\hline Polymicrobial BSI & $5(29.4)$ & $7(7.1)$ & $5.41(1.48-19.79)$ & 0.01 \\
\hline MDR BSI & $4(23.5)$ & $34(34.6)$ & $0.57(0.17-1.91)$ & 0.37 \\
\hline BSI involving Gram-positive MDR bacteria & $3(17.6)$ & $24(24.4)$ & $0.66(0.17-2.49)$ & 0.54 \\
\hline BSI involving Gram-negative MDR bacteria & $1(5.8)$ & $11(11.2)$ & $0.49(0.05-4.09)$ & 0.51 \\
\hline BSI by Escherichia coli & $11(64.7)$ & $37(37.7)$ & $3.02(1.03-8.85)$ & 0.04 \\
\hline BSI by coagulase-negative staphylococci & $4(23.5)$ & $32(32.6)$ & $0.63(0.19-2.10)$ & 0.45 \\
\hline BSI by Klebsiella pneumoniae & $3(17.6)$ & $10(10.2)$ & $1.88(0.46-7.70)$ & 0.37 \\
\hline BSI by Pseudomonas aeruginosa & $2(11.7)$ & $9(9.1)$ & $1.31(0.25-6.70)$ & 0.73 \\
\hline BSI by viridans streptococci & $3(17.6)$ & $5(5.1)$ & $3.98(0.85-18.54)$ & 0.07 \\
\hline
\end{tabular}

Data presented as $n$ (\%) unless otherwise indicated. ANC: Absolute neutrophil count; BSI: bloodstream infection; HSCT: haematopoietic stem cell transplantation; MDR: multidrug-resistant; OR: odds ratio; 95\% CI: 95\% confidence interval; SD: standard deviation. * Induction chemotherapy or HSCT;

${ }^{\dagger} \mathrm{ANC}<100$ cells $/ \mathrm{mm}^{3}$.

TABLE 4: Multiple logistic regression analysis of the risk factors for septic shock (SS) at the time of febrile neutropenia (FN) in hospitalised adult cancer patients.

\begin{tabular}{lccc}
\hline Risk factor & Adjusted OR & $95 \%$ CI & $P$ value \\
\hline & Model 1 & & \\
Polymicrobial BSI & 5.41 & $1.48-19.79$ & 0.01 \\
\hline & Model 2 & & \\
BSI by Escherichia coli & 4.30 & $1.27-14.48$ & 0.01 \\
BSI by viridans streptococci & 7.58 & $1.34-42.80$ & 0.02 \\
\hline
\end{tabular}

OR: odds ratio; $95 \%$ CI: 95\% confidence interval; BSI: bloodstream infection.

likely to develop SS at the onset of FN. In particular, BSIs involving E. coli and viridans streptococci were independently associated with SS at the time of FN. The 28-day survival rate of the patients with SS at the time of FN was significantly lower than that of the patients who did not present with SS.

Previous observational studies involving distinct populations have confirmed the influence of microbiological aspects of BSIs on the hazards of SS. Consistent with the results of our study, Leibovici et al. conducted a retrospective study involving more than 4000 episodes of bacteraemia in a general population and found that the polymicrobial aetiology was predictive of SS [11]. Moreover, the association of specific BSI by E. coli and viridans streptococci with SS in
FN patients is feasible because invasive infections by $E$. coli and viridans streptococci are often associated with significant morbidity and mortality [12-15]. For example, the study of Marron et al. [15] reported an association between viridans streptococcal bacteraemia and serious complications, such as SS and acute respiratory distress syndrome, in neutropaenic patients receiving high-dose chemotherapy with cyclophosphamide before allogeneic bone marrow transplantation.

Interestingly, none of the studied clinical characteristics was significantly associated with SS at the time of FN in the multiple logistic regression analysis. These findings differ from the immunocompetent patient studies in which the early development of SS was more frequent in the subjects with advanced age and multiple comorbidities [4-6]. One possible explanation is the relative homogeneity of our study population, which consisted of a large proportion of young patients with haematological malignancies and a relatively low prevalence of associated comorbidities. This fact highlights the need to identify the rapidly available clinical diagnostic features that can predict septic shock in this setting.

This study had some limitations. For example, we found a low incidence of bacteraemia by Staphylococcus aureus and Pseudomonas aeruginosa, which are often associated with a poorer prognosis in septic patients; therefore, our results should be interpreted with caution, as distinct virulent 
bacteria may be found in other centres. Furthermore, this study was susceptible to biases that are inherent to observational studies; however, the following factors minimised the possibility of systematic errors: the proper measurement of variables and outcomes with previously defined objective criteria, the use of standardised data collection, the implementation of a followup by a research team that was not related to the care provided, and the use of multivariate analyses.

\section{Conclusions}

The aetiology of BSIs is an important risk factor for SS at the onset of FN in adult cancer patients. Polymicrobial BSI, particularly bacteraemia by E. coli and viridans streptococci, are the risk factors for SS at the onset of FN.

Identifying the microbiological factors associated with SS in FN is of paramount importance to clinicians, as this knowledge can determine the preventative measures to avoid BSI by specific highly virulent pathogens and the best choice of empiric antimicrobial therapy.

Future studies are required to assess other possible risk factors for the early onset of SS in the context of FN and to determine whether specific interventions based on the early identification of highly virulent bacteria could result in an effective method to prevent SS and its characteristically pronounced mortality rates.

\section{Conflict of Interests}

The authors declare no conflict of interests. This work was partially supported by CNPq (Brazilian National Council of Research).

\section{Acknowledgments}

The authors thank the patients, the data collection team who created the database, and the Hospital de Clínicas de Porto Alegre, particularly the haematology division and the Infection Control Committee, for their support in conducting the study.

\section{References}

[1] M. Legrand, A. Max, V. Peigne et al., "Survival in neutropenic patients with severe sepsis or septic shock," Critical Care Medicine, vol. 40, no. 1, pp. 43-49, 2012.

[2] I. Malik, M. Hussain, and H. Yousuf, "Clinical characteristics and therapeutic outcome of patients with febrile neutropenia who present in shock: need for better strategies," Journal of Infection, vol. 42, no. 2, pp. 120-125, 2001.

[3] A. Lever and I. Mackenzie, "Sepsis: definition, epidemiology, and diagnosis," British Medical Journal, vol. 335, no. 7625, pp. 879-883, 2007.

[4] D. C. Angus and T. van der Poll, "Severe sepsis and septic shock," The New England Journal of Medicine, vol. 369, no. 9, pp. 840851, 2013.
[5] J. P. Quenot, C. Binquet, F. Kara et al., “The epidemiology of septic shock in French intensive care units: the prospective multicenter cohort EPISS study," Critical Care, vol. 17, no. 2, article R65, 2013.

[6] J. L. Vincent, Y. Sakr, C. L. Sprung et al., "Sepsis in European intensive care units: results of the SOAP study," Critical Care Medicine, vol. 34, no. 2, pp. 344-353, 2006.

[7] A. S. Cross, "What is a virulence factor?" Critical Care, vol. 12, article 196, 2008.

[8] CLSI, "Performance standards for antimicrobial susceptibility testing: twenty-second informational supplement," CLSI Document M100-S22, Clinical and Laboratory Standards Institute, Wayne, PA, USA, 2012.

[9] WHO, Handbook for Reporting Results of Cancer Treatment, World Health Organization, Geneva, Switzerland, 1979.

[10] M. M. Levy, M. P. Fink, J. C. Marshall et al., "SCCM/ESICM/ ACCP/ATS/SIS international sepsis definitions conference," Critical Care Medicine, vol. 31, no. 4, pp. 1250-1256, 2003.

[11] L. Leibovici, M. Drucker, H. Konigsberger et al., "Septic shock in bacteremic patients: risk factors, features and prognosis," Scandinavian Journal of Infectious Diseases, vol. 29, no. 1, pp. 71-75, 1997.

[12] J. Rodríguez-Baño, J. Mingorance, N. Fernández-Romero et al., "Outcome of bacteraemia due to extended-spectrum $\beta$-lactamase-producing Escherichia coli: impact of microbiological determinants," Journal of Infection, vol. 67, no. 1, pp. 27-34, 2013.

[13] A. R. Tunkel and K. A. Sepkowitz, "Infections caused by viridans streptococci in patients with neutropenia," Clinical Infectious Diseases, vol. 34, no. 11, pp. 1524-1529, 2002.

[14] Y. Okamoto, R. C. Ribeiro, D. K. Srivastava, J. L. Shenep, C. H. Pui, and B. I. Razzouk, "Viridans streptococcal sepsis: clinical features and complications in childhood acute myeloid leukemia," Journal of Pediatric Hematology/Oncology, vol. 25, no. 9, pp. 696-703, 2003.

[15] A. Marron, J. Carratalà, E. González-Barca, A. Fernández-Sevilla, F. Alcaide, and F. Gudiol, "Serious complications of bacteremia caused by viridans streptococci in neutropenic patients with cancer," Clinical Infectious Diseases, vol. 31, no. 5, pp. 11261130, 2000. 

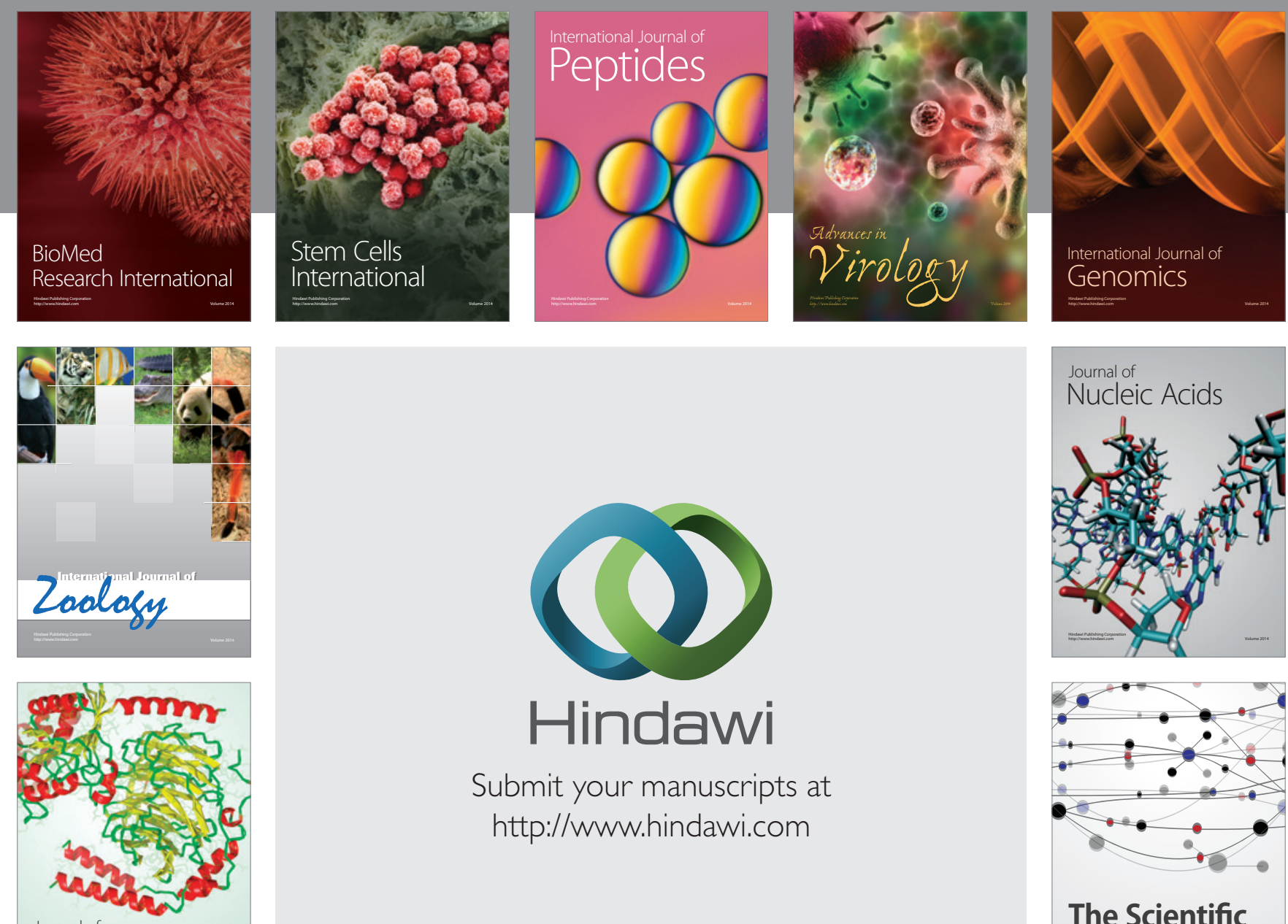

Submit your manuscripts at

http://www.hindawi.com

Journal of
Signal Transduction
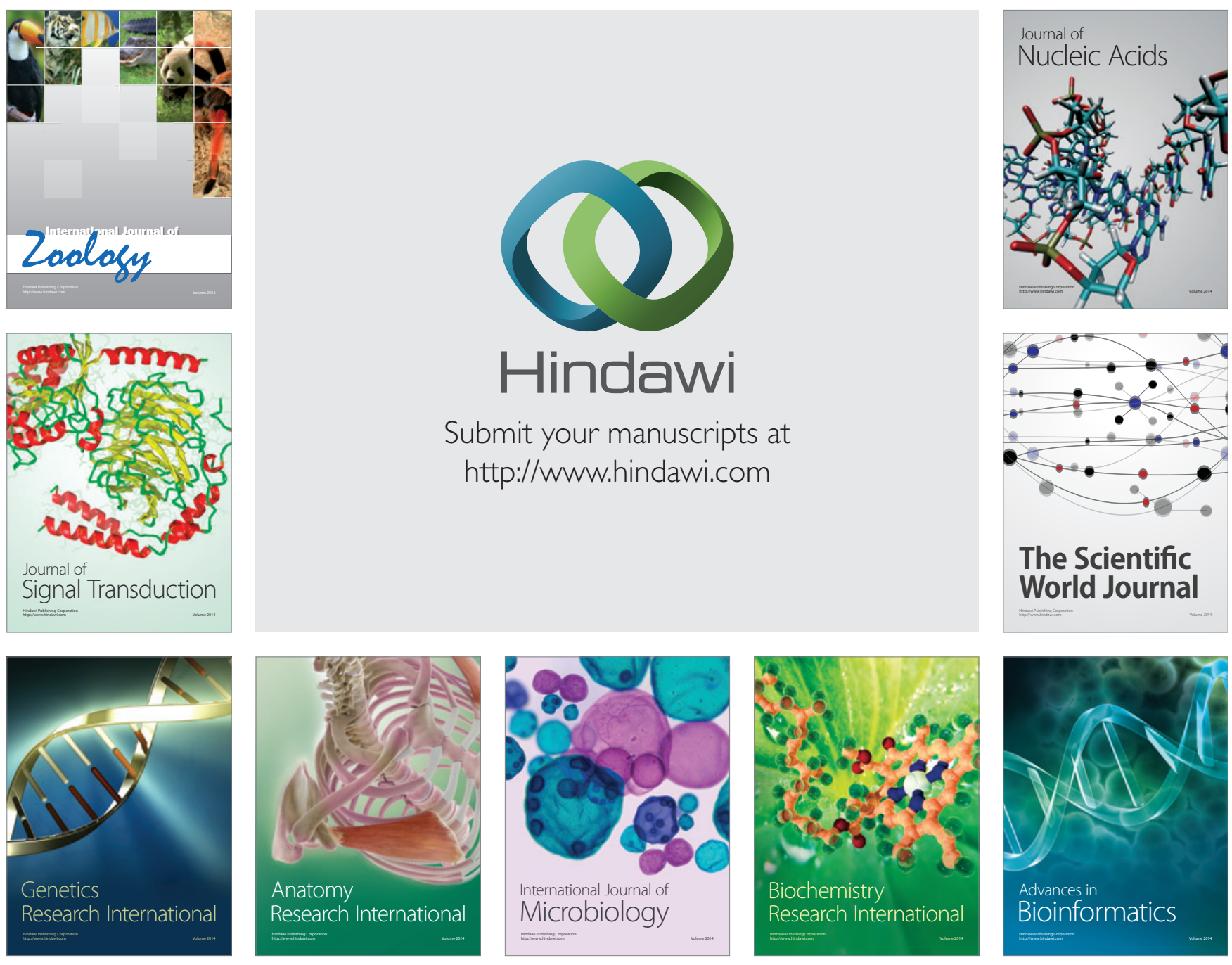

The Scientific World Journal
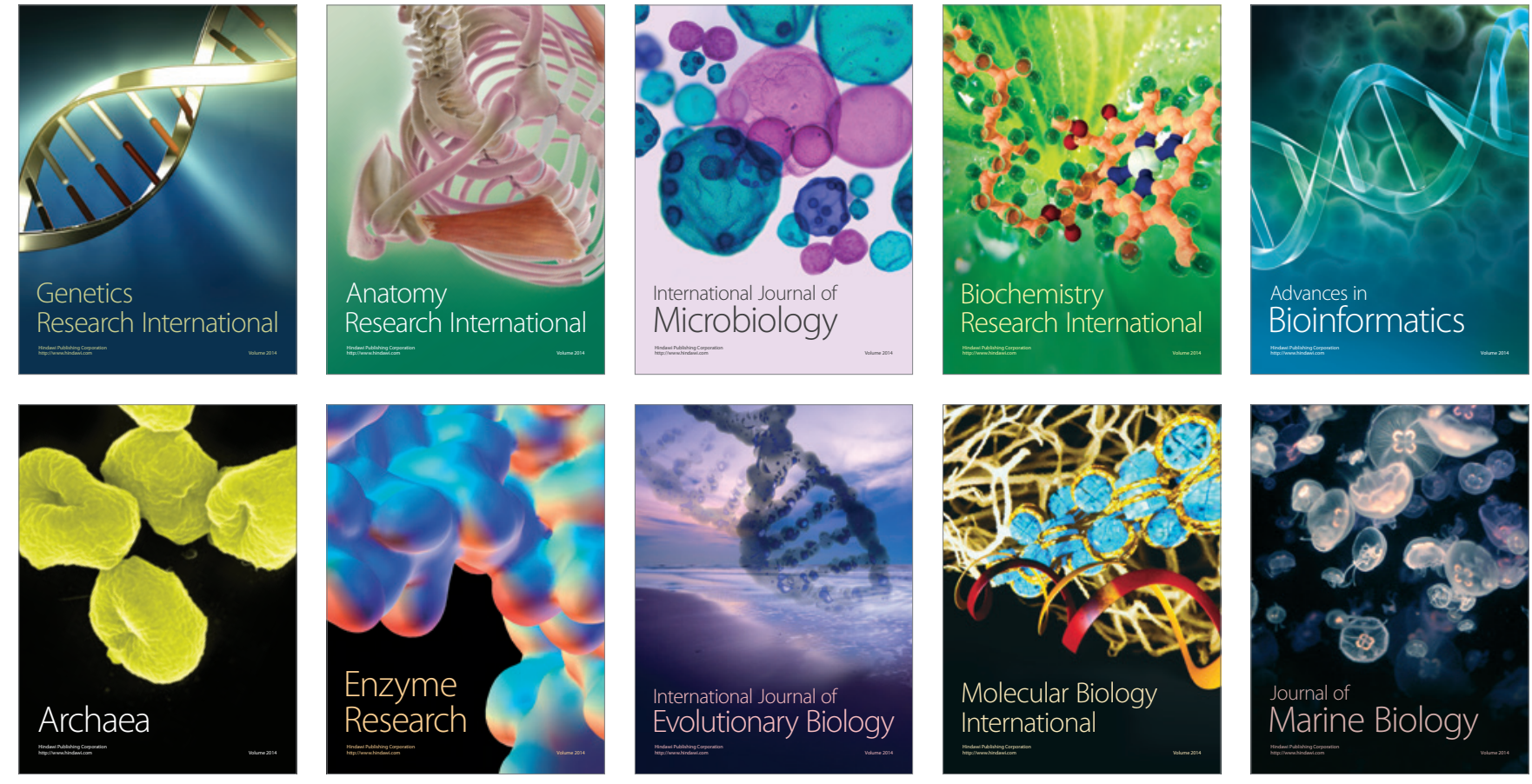